\title{
IMPACT OF THE QUALITY OF MEASUREMENT RESULTS ON CONFORMITY ASSESSMENT
}

\author{
Biserka Runje, Amalija Horvatić Novak \& Zdenka Keran
}
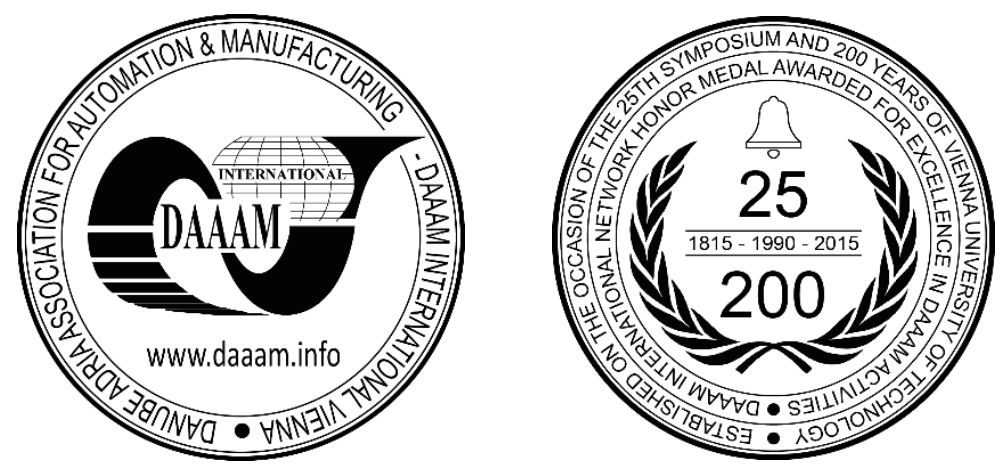

This Publication has to be referred as: Runje, B[iserka]; Horvatic Novak, A[malija] \& Keran, Z[denka] (2018). Impact of the Quality of Measurement Results on Conformity Assessment, Proceedings of the 29th DAAAM International Symposium, pp.0051-0055, B. Katalinic (Ed.), Published by DAAAM International, ISBN 978-3-902734-20-4, ISSN 1726-9679, Vienna, Austria

DOI: $10.2507 / 29$ th.daaam.proceedings.007

\begin{abstract}
It is well known that measurement uncertainty is a key indicator of the quality of measurement results. We evaluate measurement uncertainty because the measurements are not perfect, for the purpose of unambiguous expression and comparison of measurement results obtained in various calibration and testing laboratories and also in order to compare the measurement results with the manufacturer's specifications or tolerance interval. In the paper, the impact of measurement uncertainty on the acceptance or rejection of a product according to its specification and measurement result is analysed. Decision rule based on simple acceptance and decision rules based on guard bands are described. In order to determine whether specified requirements relating to a product, process or system are fulfilled the different methods for uncertainty evaluation are also discussed.
\end{abstract}

Keywords: measurement uncertainty; conformity assessment; producer's risk; consumer's risk; decision rules

\section{Introduction}

Through the adoption of the 1993 International Agreement on the Expression of Uncertainty in Measurement, it is now possible to unequivocally express and compare measurement results obtained in various institutes as well as measurement and test laboratories. In accordance with the Guide to the Expression of Uncertainty in Measurement (GUM) measurement uncertainty is defined as a parameter associated with the result of a measurement that characterizes the dispersion of the values that could reasonably be attributed to the measurand [1]. Although great emphasis is placed upon the GUM method, primarily due to the fact that it provides a framework which enables a consistent calculation of uncertainty for random and systemic actions, it was soon demonstrated that the application of the GUM method in certain cases in which the preconditions required by the method were not met led to unacceptable results.

If the functional relationship between output quantity $y$ and its input quantities is nonlinear and a first-order Taylor expansion of the relationship is not an acceptable approximation, then the probability distribution of $y$ cannot be obtained by convolving the distributions of the input quantities. In such cases, other analytical or numerical methods are required [2]. 
In 1997, seven international organisations which drafted the original version of the GUM, founded the Committee for Joint Guidelines in Measurement (JCGM) with the stated goal of promoting the use of the GUM as well as the preparation of the amendments to the original version of the GUM necessary for its wider application. Since that time the Committee for Joint Guidelines in Measurement has issued four supplements to the GUM with three more supplements as well as a revised version of the guide still to be issued in the future. There currently exist no guidelines in the GUM or other JCGM documents for resolving the issues of the proper application of the principles of measurement uncertainty in Metrology.

The metrology community is aware of this problem and is currently engaged in solving it through updating the existing documents as well as through developing models designed to facilitate the practical implementation of such guidelines. In accordance with the suggested revision of the GUM titled „JCGM 100201 X CD“ the simplest way to overcome the aforementioned difficulties and deficits of the GUM is to introduce the Bayesian approach and apply the Monte Carlo Simulation (MCS) in the procedure of calculating measurement uncertainty. [3].

Furthermore, in order to overcome the aforementioned issues it is vitally important to develop new models for evaluating measurement uncertainty which would allow the harmonised application and implementation of measurement uncertainty principles as well as reliable decision making on the complementarity of the results with the given specifications. Conformity assessment, as broadly defined, is any activity undertaken to determine, directly or indirectly, whether a product, process, system, person or body meets relevant standards and fulfils specified requirements. In conformity assessment, a measurement result is used to decide if an item of interest conforms to a specified requirement. In a typical situation, the needs and wishes of the consumer have to be balanced against the capabilities and promises of the producer (supplier). [4].

\section{The role of measurement uncertainty in conformity assessment}

Measurement results are most commonly expressed through arithmetic means which is considered to be the best estimation of the measurand as well as measurement uncertainty with an associated coverage probability. A measurement result may also be expressed by giving a coverage interval for the measurand with an associated coverage probability, by a probability density function (PDF) or by a numerical approximation to a PDF. In most cases a normal PDF is assumed. $[5]$.

In accordance with the ISO 14253-1 [6], in the case of a two-sided tolerance interval, the guarded acceptance decision rule for demonstrating conformity with specifications is presented in Figure 1. Assuming that the true value of a measurable property lies within a tolerance interval and provided that the measured value of the property lies within the acceptance interval the item will be accepted as conforming. Measurement result is given by a normal probability density function.

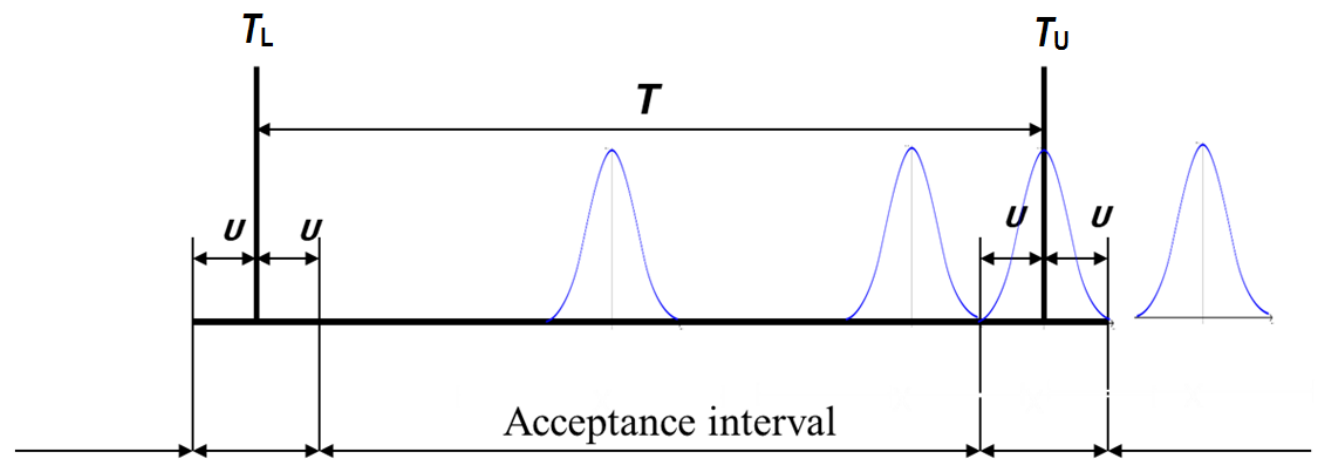

Fig. 1. The guarded acceptance decision rule for demonstrating conformity with specifications

Where:

$T_{\mathrm{L}}-$ lower specification limit

$T_{\mathrm{U}}$ - upper specification limit

$T$ - tolerance interval

$U$ - expanded measurement uncertainty.

If the expanded measurement uncertainty $U$ is expressed as a product of the standard measurement uncertainty $u$ and the coverage factor $k$ ( $k=2$ in this case), for any measured value lying within the acceptance interval, the maximum probability of accepting a non-conforming item will be $2.3 \%$, assuming a normal PDF for the measured quantity. The decision to accept an item as conforming or reject it as non-conforming according to the specifications, is based on the measured values of object characteristics in relation to the acceptance criteria. An acceptance limit outside a tolerance interval can also be chosen so as to increase the probability that a rejected item is truly non-conforming. 
Such a guarded rejection decision rule is often employed when one wants clear evidence that a limit has been exceeded prior to taking a negative action [6]. With a decision rule based on simple acceptance and with the measured value given by a normal distribution lying at the lower or upper specification limit, the probability of accepting or rejecting a conforming or non-conforming item (Figure 2) will be $50 \%$. When trying to conclude if the item is accepted or rejected there would be a $50 \%$ chance of an incorrect decision.

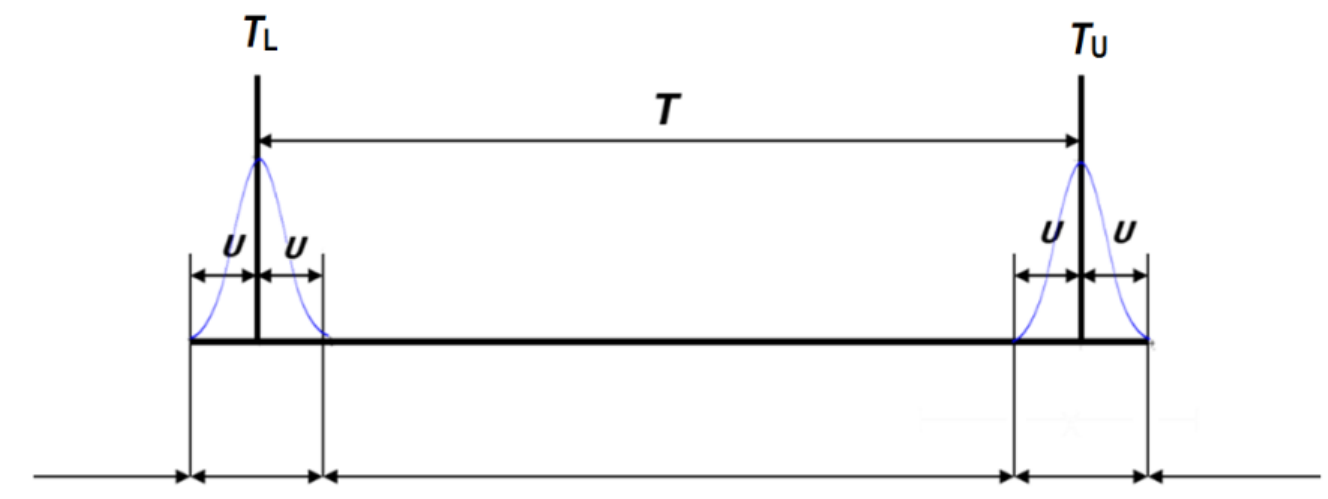

Fig. 2. Simple acceptance decision rule at lower and an upper tolerance limits $T_{\mathrm{L}}$ and $T_{\mathrm{U}}$

Due to the uncertainty in the measurement, there is always the risk of making incorrect decisions (Figure 3). There are four types of decisions: a) an item accepted as conforming may actually be conforming, b) an item accepted as conforming may actually be non-conforming, c) an item rejected as non-conforming may actually be conforming and d) an item rejected as non-conforming may actually be conforming.

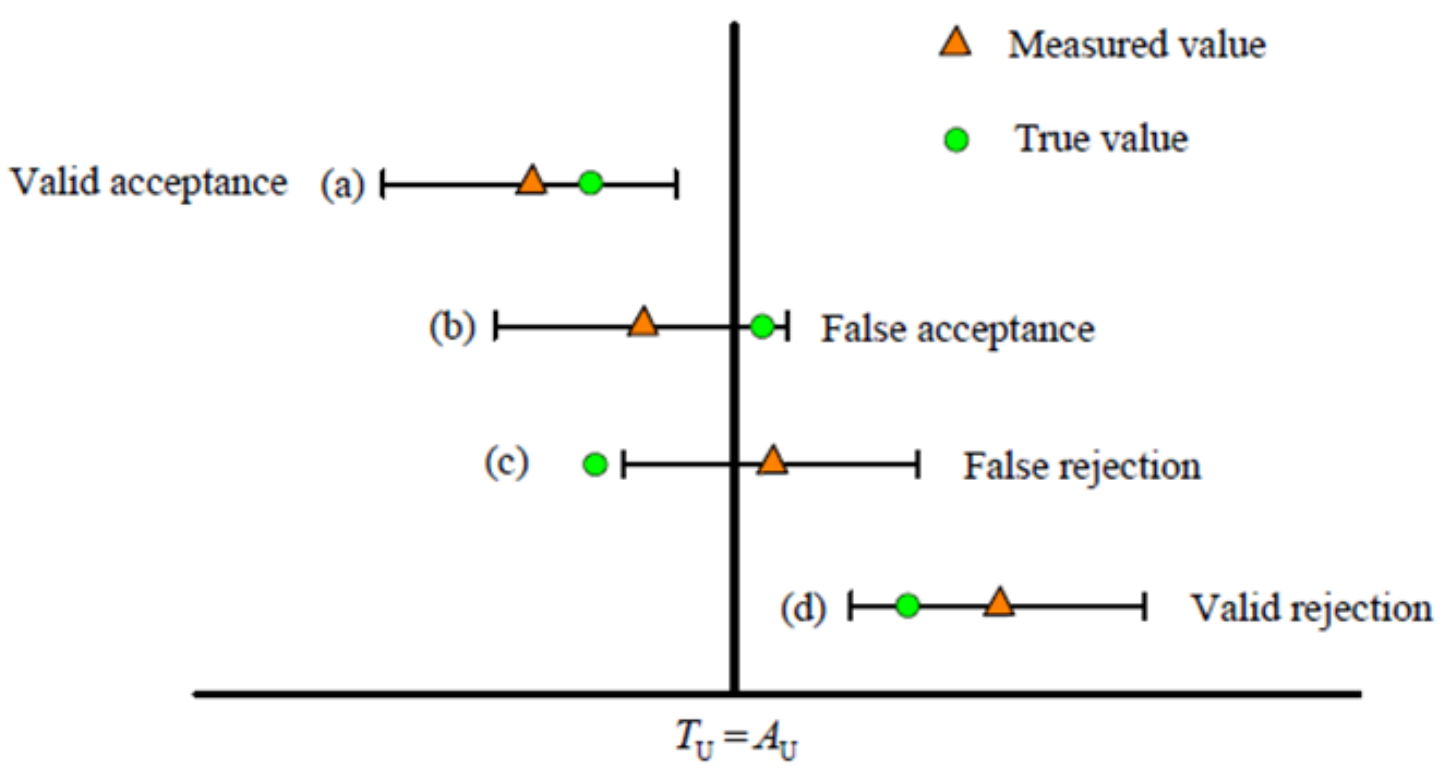

Fig. 3. Simple acceptance decision rule near an upper tolerance limit $T_{\mathrm{U}}, A_{\mathrm{U}}=T_{\mathrm{U}}[5]$

Where:

$A_{\mathrm{U}}$ - upper acceptance limit

$T_{\mathrm{U}}$ - upper specification limit.

The risk of accepting a non-conforming item can be reduced by establishing an upper acceptance limit $A_{\mathrm{U}}$ inside the tolerance interval. An acceptance limit placed outside the tolerance interval can be selected in order to increase the probability that the rejected item is non-conforming. Choosing the tolerance and acceptance intervals are political and business decisions which depend upon factors linked to potential deviations from planned product quality. Generally, the decision to accept an item as conforming or to reject it as non-conforming will also depend on the number of measured characteristics. Additionally, taking into account the fact that each of these characteristics has a defined specification limit, many different acceptance and rejection decision can be made. 
Therefore, it is especially important to correctly estimate the measurement uncertainty so that the "true" value is expected to be within the uncertainty range and the evaluated measurement uncertainty is realistic. In some cases, e.g. deviations of roundness or roughness are always positive with measured values close to zero, the confidence interval evaluated according to the current GUM is clearly not reliable because it includes infeasible (negative) values of the output $y$. [7]. In such cases, other analytical or numerical methods are required.

The use of the GUM and Monte Carlo methods (MCS) for the output quantity $y$, which is described using the equation $y=x^{2}$, is shown in Figure 4. The input quantity is defined by the Gaussian probability density function with the mean being 3 and the standard deviation being 5 . Figure 4 shows the probability density functions of the output quantity $y=x^{2}$ obtained using the GUM method and Monte Carlo simulation (MCS) with the number of the conducted simulations being $M=100000$. It is clearly visible that the confidence interval evaluated according to the current GUM is not reliable because it includes infeasible (negative) values of the output $y$.

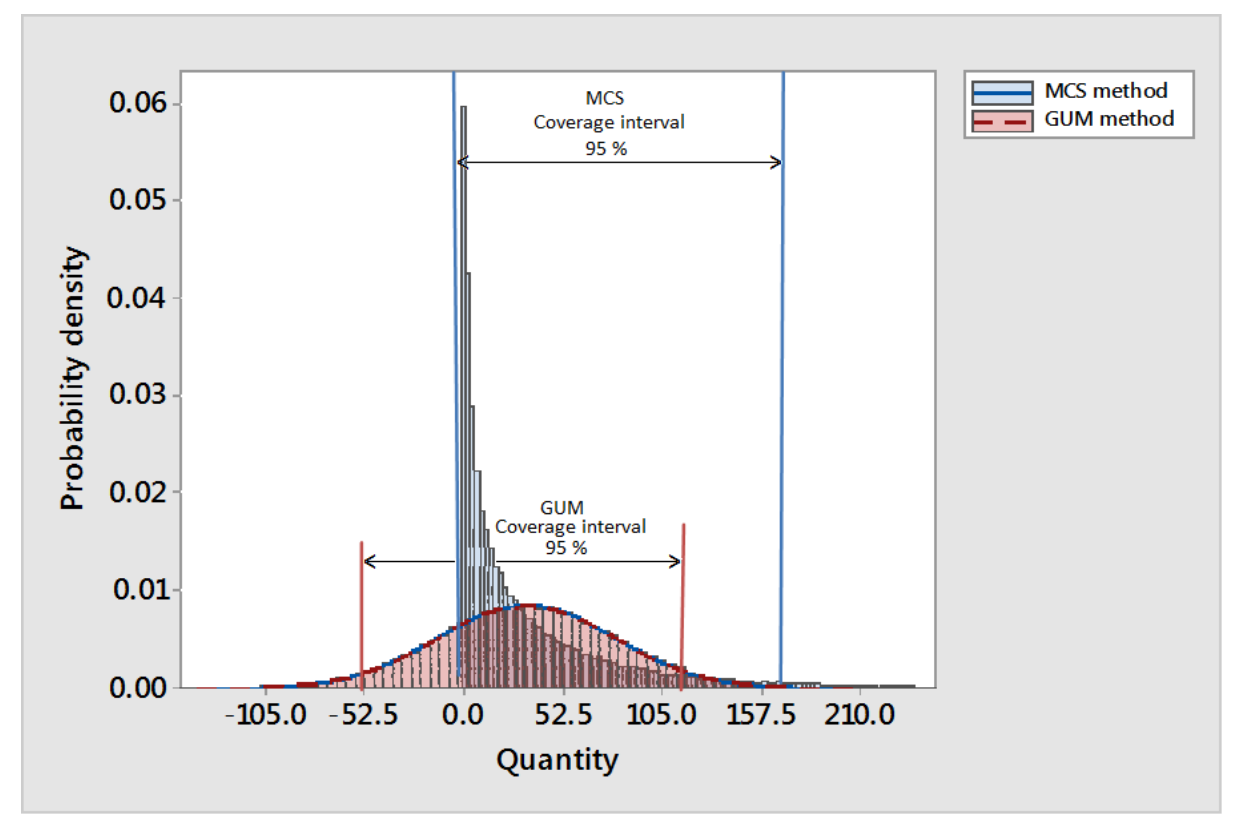

Fig. 4. Probability density functions and coverage intervals for output quantity $y=x^{2}$

The decision to accept the item as conforming or reject it as non-conforming is based on the measured value of a characteristic of the item as referenced with the applied decision rule. The aim of risk analyses is to compare the parameters of a real/designed product with specification limits. Those parameters which do not meet the criteria, in other words parameters which transcend specification limits, must be rearranged. [8]. Measurement uncertainty is one of the factors which must be taken into consideration when defining the acceptance criteria. The acceptance or rejection of an item when the measured value of its examined characteristic lies close to the tolerance limit can result in an incorrect decisions being made as well as lead to unintended consequences. An important and widely used „decision rule“ is that of ,simple acceptance“ or ,shared risk“. Under such a rule, the producer and the user (consumer) accept or reject an item as conforming or non-conforming based on whether its measurement result lies within tolerance limits. Just as the name „shared risk“ implies, using the „,decision rule“ the producer and consumer share the consequences of incorrect decisions. In practice, in order to keep the probability of making incorrect decisions in acceptable margins, it is necessary for there to exist a consensus between the producer and the consumer that the measurement uncertainty should be considered and deemed acceptable for the intended purpose of the product.

\section{Conclusion}

Measurement uncertainty is one of the factors which must be taken into consideration when defining the acceptance criteria. The acceptance or rejection of an item when the measured value of its examined characteristic lies close to the tolerance limit can result in an incorrect decisions being made as well as lead to unintended consequences. Relying on the ,simple acceptance decision rule“ and the measured value given by a normal distribution can lead to the probability of accepting a non-conforming item or the rejection of a conforming item to be as high as $50 \%$. It is especially important to correctly estimate the measurement uncertainty so that the "true" value is expected to be within the uncertainty range and the evaluated measurement uncertainty is realistic. Furthermore, in order to overcome the aforementioned issues it is vitally important to develop new models for evaluating measurement uncertainty which would allow for the harmonised application and implementation of measurement uncertainty principles as well as reliable decision making on the complementarity of results with the given specifications. 


\section{References}

[1] JCGM 100:2008 GUM 1995 with minor corrections Evaluation of measurement data — Guide to the expression of uncertainty in measurement

[2] JCGM 101:2008 Evaluation of measurement data - Supplement 1 to the "Guide to the expression of uncertainty in measurement" - Propagation of distributions using a Monte Carlo method

[3] Lira, I.; (2016). The GUM revision: the Bayesian view toward the expression of measurement uncertainty, European Journal of Physics, Vol 37, No 2, 2016, pp 1-16

[4] Pendrill, L. R.; (2014). Using measurement uncertainty in decision-making and conformity assessment, Metrologia Vol 51, No 4, 2014, pp. 206-218

[5] JCGM 106:2012 Evaluation of measurement data - The role of measurement uncertainty in conformity assessment

[6] ISO 14253-1:2017 - Geometrical product specifications (GPS) - Inspection by measurement of workpieces and measuring equipment - Part 1: Decision rules for verifying conformity or nonconformity with specifications.

[7] Runje, B.; Horvatic Novak, A.; Alar, V.; Medic, S. \& Bosnjakovic, A. (2016). Examples of measurement uncertainty evaluations in accordance with the revised GUM, Journal of Physics: Conference Series Metrology across the Sciences: Wishful Thinking?, University of California, Berkeley, California, 9781510835290, Wilson, Met al. (eds), pp. 38-44, Institute of Physics Publishing, Berkeley, California

[8] Broum, T; Dvorak, J \& Kleinova, J. (2011). Value optimization and risks elimination of product, Annals of DAAAM for 2011 \& Proceedings of the 22nd International DAAAM Symposium, Volume 22, No. 1, ISSN 1726-9679 ISBN 978-3-901509-83-4, Katalinic, B. (Ed.), pp. 757-758, Published by DAAAM International, Vienna, Austria, EU 